\title{
Qualitätsmanagement im Dienstleistungssektor ISO 9000 und Dienstleistungen
}

\section{Ueli Burkhard*}

Gegründet 1828 als Schiffs-Klassifikationsgesellschaft hat Bureau Veritas $(B V)$ in seiner langen Geschichte eigentlich immer etwas mit Qualität oder Qualitätssicherung zu tun gehabt. Heute ist Bureau Veritas in 125 Ländern der Welt mit 500 Service-Zentren vertreten.
Seit 1987 befasst sich Bureau Veritas Quality International (BVQI) als $100 \%$ Tochter von Bureau Veritas mit der Beurteilung von Qualitätssicherungs-Systemen, im Grunde genommen eine logische Weiterentwicklung. Weltweit hat BVQI bis heute $c a$. 1000 Unternehmen zertifiziert.

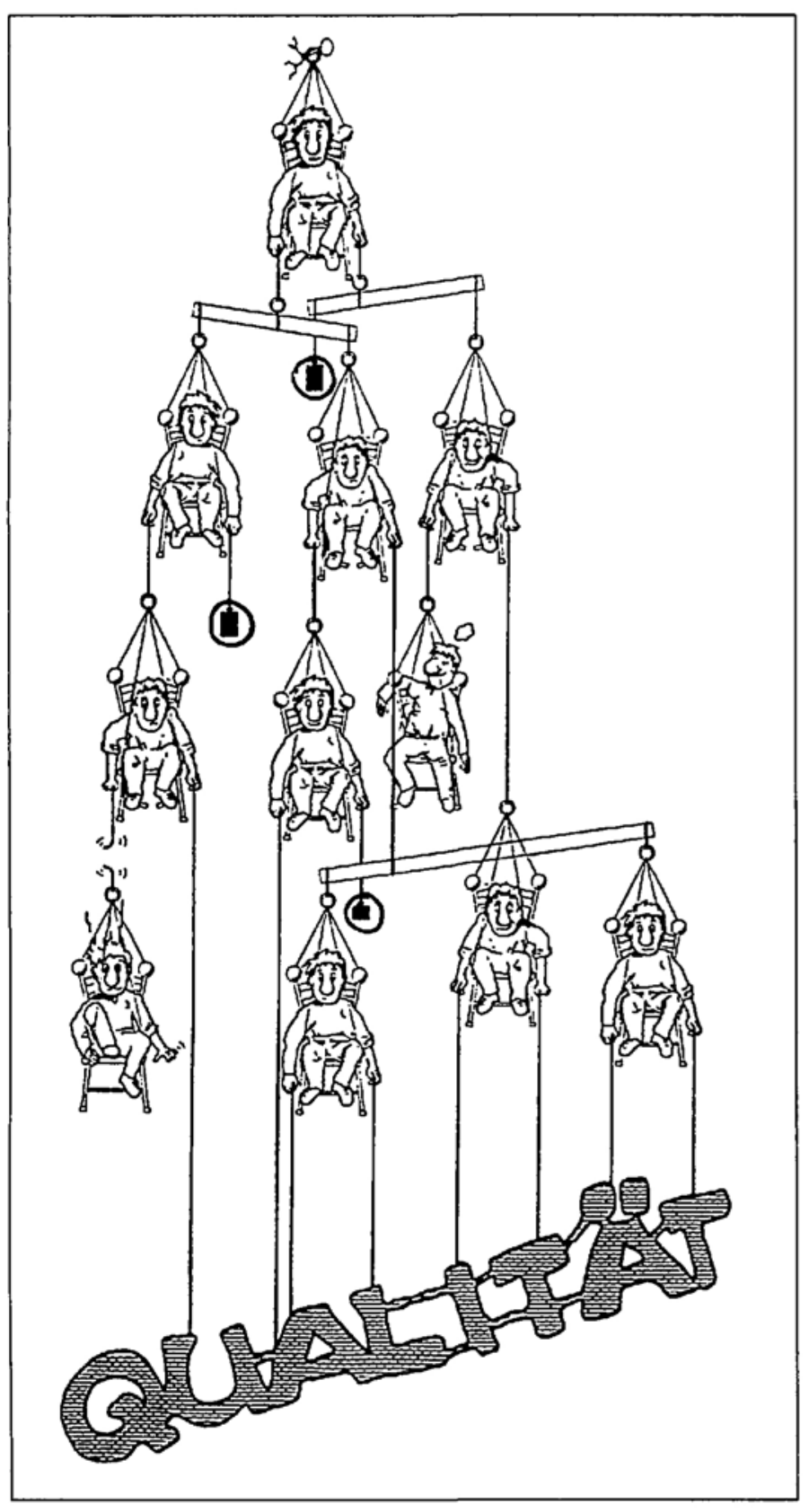

Fig. 1. Bureau Veritas Quality International

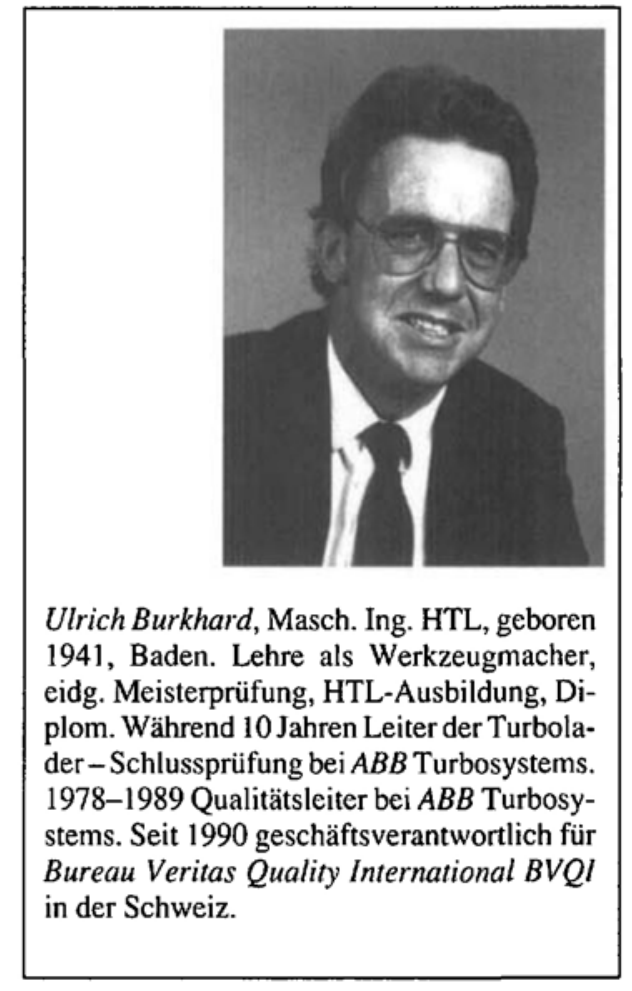

1990 nahm $B V Q I$ seine Tätigkeit auch in der Schweiz auf. Die Überprüfung von $B V Q I$ durch die schweizerische Akkreditierungsstelle auf der Basis der europäischen Norm EN 45012 verlief erfolgreich, die Akkreditierung durch die zuständige Behörde steht kurz bevor.

Mein Referat möchte ich mit dem Bild eines Mobiles (Fig. 1) mit vielen ausbalancierten Einzel-Elementen beginnen. Ich möchte damit aufzeigen wie wichtig es ist, dass eine Organisation harmonisch aufeinander abgestimmt ist. Wenn irgend ein Element unserer Organisation oder unseres QS-Systemes nicht auf das andere abgestimmt ist, dann stimmt eben etwas nicht oder anders gesagt unsere Organisation hängt schief. Ich glaube, dass ein so ausbalanciertes Gebilde wie ein Mobile sowohl für ein Fabrikations- als auch ein Dienstleistungs-Unternehmen seine Gültigkeit hat. Nebenbei gesagt haben ja viele Elemente in einem Produktionsbetrieb ebenfalls Dienstleistungsaufgaben, ich denke da an Funktionen wie Einkauf, Administration, Unterhalt usw.

Was heisst QS im Dienstleistungsbereich?

In vielen Gesprächen mit Kunden, Freunden und Kollegen kann ich immer wieder feststellen, dass zwar jedermann von Dienstleistungs-Qualität spricht, kaum

*Korrespondenz: U. Burkhard
Bureau Veritas
Badenerstrasse 780
Postfach
CH-8048 Zürich


jemand weiss aber was darunter zu verstehen ist.

Kürzlich ging es anlässlich eines Weiterbildungseminares in einem Ingenieurunternehmen darum sämtlichen Mitarbeiterinnen und Mitarbeitern von der Telefonistin bis zum Geschäftsleiter klar zu machen, was ISO 9001 umgesetzt auf dieses Unternehmen zu bedeuten hat. Jede Mitarbeiterin und jeder Mitarbeiter hatten zwei persönliche Antworten zu geben was Qualität am Arbeitsplatz bedeutet, welche Elemente für sie/ihn am Wichtigsten sind. Die Antworten wurden durch uns auf die Norm ISO 9001 referenziert (Fig. 2).

Berücksichtigt man auch noch die Tatsache, dass die QS Norm ISO 9001 einige spezifische QS-Elemente regelt, ich denke da an interne Audits, wurden sämtliche Elemente der Norm abgedeckt.

Mit dieser Aussage kann auch beantwortet werden, in welchen Bereichen eines Dienstleistungs-Unternehmen ISO 9000 angewendet werden kann: nämlich in allen.

Die Umsetzung der Norm ISO 9000 in die Praxis

Die Anpassung einer Dienstleistunsorganisation an eine QS-Norm wie z.B. ISO 9002/EN 29 002, bietet erfahrungsgemäss Schwierigkeiten, weil nicht klar ist auf welche 'Messlatte' die Qualitätssicherung eingestellt werden muss. Gerade im Dienstleistungsbereich ist häufig auch nicht klar was mit Prozessbeherrschung, Einkauf oder Identifikation und Rückverfolgbarkeit gemeint ist.

Eine weitere Schwierigkeit besteht darin, dass die 'Fremdsprache' ISO 9000 in der internen Sprache des Unternehmens nicht verstanden wird und demzufolge nicht zweckmässig umgesetzt werden kann.

Im folgenden möchte ich Ihnen einige Beispiele von Stärken und Schwächen von Dienstleistungs-Unternehmungen vor der Einführung eines strukturierten QS-Systemes geben:

Umsetzung der Norm ISO $9000 \mathrm{im}$

Dienstleistungssektor

Transportunternehmen

+ Guter Teamgeist, vorbildliches TQM durch eine effiziente und mitarbeitergerechte Organisation.

- Unvollständige Dokumentationen auf allen Ebenen des Unternehmens.

Headquarter eines multinationalen Unternehmens

+ Effiziente und sehr umfassendes EDVSystem als erstklassiges TQM und QSInstrument.
4.1 Verantwortung der obersten Leitung

4.2 Qualitätssicherungssystem

4.3 Vertragsüberprügung

4.4 Designlenkung

4.5 Lenkung der Dokumente

4.6 Beschaffung

4.7 Vom Auftraggeber beigestellite Produkte

4.8 Identifikation und Rückverfolgbarkeit

4.9 Prozesslenkung

4.10 Prüfungen

4.11 Prüfmittel

4.12 Prüfstatus

4.13 Lenkung fehlerhafter Produkte

4.14 Korrekturmassnahmen

4.15 Handhabung Lagerung und Versand

4.16 Qualitätsaufzeichnungen

4.17 Interne Qualitätsaudits

4.18 Schulung

4.19 Kundendienst

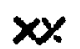

$x \times x$

$x \times x \times$

$x \times \times \times \times x \times$

$x \times x \times x$

$x$

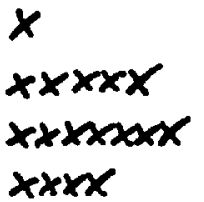

$x \times \times x$
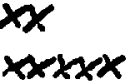

$X$

\section{C}

ig. 2. Betriebsgerechte Umsetzung der ISO 9000 (BVQU)

- Unklare Aufgabenabgrenzungen, viele Spielregeln des Unternehmens nur in einzelnen Köpfen.

Grosshandelsunternehmen

+ Ebenfalls eine sehr effiziente und flexible EDV, sozusagen papierloses Unternehmen.

- Schwierig, Belange der Schulung in diesem vielschichtigen Unternehmen unter einen Hut zu bringen.

Generell in diesen Unternehmungen:

Viele Direktinformationen bzw. mündliche Festlegungen in den Köpfen (Müller weiss nicht was Meier tut).

TQM oder QS im Dienstleistunsunternehmen ist zwar relativ neu als Begriff, es zeigt sich aber auch hier einmal mehr, dass bei einem gut geführten Unternehmen auch ohne Begriffe wie ISO 9000 oder TQM wesentliche Elemente eines normgerechten QS-Systemes bereits vorhanden sind.

An einigen Beispielen möchte ich Ihnen aufzeigen, was eine Umsetzung der Norm in die Praxis heissen kann.

Branchenbezogene Umsetzung einzelner QS-Elemente fung

ISO 9001 PKT. 4.3 Vertragsüberprü-

Consulting

Abfassen eines Dienstleistungskontraktes (Was will jeder)

\section{Grosshandel:}

Sicherstellen, dass der richtige Artikel durch korrektes Umsetzen der Kundenbestellung in die interne Sprache sichergestellt wird (Tel, Fax, EDV, Kataloge) kumente

Consulting:

Sicherstellen, dass nur aktuelle Kundendokumente verwendet werden

Grosshandel:

Sicherstellen, dass Angaben in Verkaufskatalogen und im EDV-System laufend aktualisiert werden

ISO 9001 PKT. 4.9 Prozesslenkung

Consulting:

Regelmässiger SOLL-IST-Vergleich mit den Vertragsgrundlagen

(Projektlenkung)

Grosshandel:

Sicherstellen, dass die Lagerorganisation den hohen Anforderungen genügt.

Probleme beim Aufbau eines QS-Systems

Der Angelpunkte sind viele. Erfahrungsgemäss sehe ich beim Aufbau und der Einführung von QS-Systemen im Dienstleistungssektor folgende Hauptschwierigkeiten:
ISO 9001 PKT. 4.5 Lenkung der Do- 
- Was heisst ISO 9000 für mein Unternehmen?

- Unklare oder keine Anweisungen an den einzelnen Arbeitsplätzen

- Lösungen werden zu weit weg gesucht, naheliegendes wird vergessen

- Die Geschäftsleitung steht nicht hinter dem Aufbau, eine effiziente Aufbauarbeit wird dadurch fraglich

- Die Aufbauarbeit wird durch eine Person gemacht, die Betroffenen stehen nicht dahinter

- Ungeeignete Berater oder unklare Zielsetzungen können bei der Einführung eines QS-Systemes zu hohen Kosten und/oder Misserfolg führen.

Nur gezieltes, klares und konsequentes Vorgehen führt zum Ziel oder zur Zertifizierung.

\section{Nutzen eines integrierten}

QS-Systemes im Dienstleistungssektor

Klare Abläufe, Aufgabenabgrenzungen und zweckmässige Arbeitsinstruktionen sind, im Grunde genommen, die recht banalen Grundlagen eines effizienten integralen QS-Systemes.

Gerade das Festlegen und beachten strukturierter Abläufe oder besser gesagt Spielregeln, profiliert das DienstleistungsUnternehmen gegenüber seiner Konkurrenz.

Es gibt zwei Hauptgründe, warum sich ein Dienstleistungs-Unternehmen zertifizieren lassen will/muss.

- Die Kunden verlangen es auch von Dienstleistungs-Zulieferanten

- Das Dienstleistungs-Unternehmen will sich gegenüber seiner Konkurrenz durch Zertifizierung profilieren

\section{Kann TQM oder ISO 9000 'verkauft' werden?}

Leider ist es auf dem Gebiete der QS schwierig, konkrete Aussagen über deren Wirtschaftlichkeit zu machen. Zuviele nur schwer erfassbare Einflüsse spielen da mit hinein.

Verbesserungen in der Aufbau- und Ablauforganisation sind Resultate einer eingeführten QS-Organisation.

Erfahrungen zeigen, dass Investitionen in QS-Systeme in kurzer Zeit amortisiert werden können.

Wie bereits angetönt gibt es verschiedene Zertifizierungs-Argumente:

- Know-how Erhaltung

- Das Dienstleistungsunternehmen will es

- Abbau yon Handelsbarrieren

- Motivationsfaktor für Mitarbeiter
- Zertifizierung als Marketing Instrument

- Der Kunde verlangt es

Im Gespräch mit einem Unternehmer des Dienstleistungs-Sektors wurde mir auf die Frage - was war der Nutzen der ihnen der Aufbau und die Zertifizierung ihrer Organisation nach ISO 9002 gebracht hat - folgende Antwort gegeben:

- Klarere organisatorische Abläufe

- Bessere Transparenz

- Zielgerichtetere Schulung

- 'Ich' weiss besser was im Markt passiert

- Ein weiteres grosses Rationalisierungs-Potential

\section{Die Entwicklung aus Sicht $B V Q I$}

- QS auf dem sehr schwierigen Gebiete der Software ist zunehmend ein Thema
- Europa- bzw. weltweit wird QS bei den Spediteuren und Transporteuren aktuell

- Im Bausektor beginnt sich einiges zu bewegen

- Versicherungen sehen in ISO 9000 ebenfalls grosse Möglichkeiten, Versicherungsrisiken zu optimieren

- Banken zeigen starkes Interesse an ISO 9000

- British Mail baut in einigen Bereichen normgerechte QS-Systeme auf.

Auch im Dienstleistungsbereich wird Qualitätssicherung, ISO 9000, Zertifizierung und TQM zunehmend zum sehr wichtigen Thema.

Die Erkenntnis setzt sich auch in dieser Branche auf einer breiten Basis durch, dass es sich lohnt und sich sehr bezahlt macht sich mit TQM und ISO 9000 auseinander zu setzen (Fig. 3).

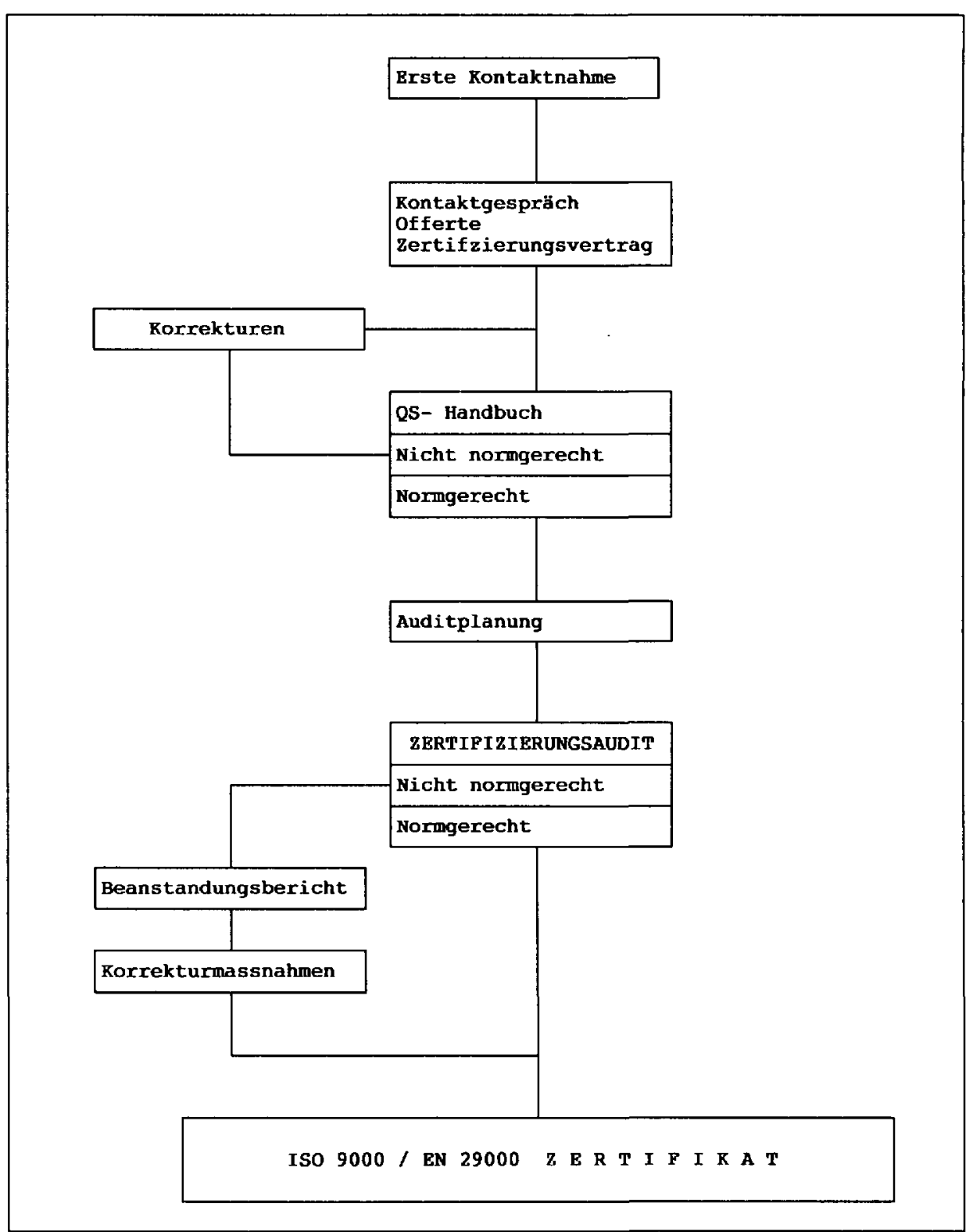

Fig. 3. Ablauf einer BVQI-Zertifizierung 